\title{
ALCALÓIDES PIRROLIZIDÍNICOS EM ESPÉCIES DO GÊNERO Senecio
}

\section{Chana de Medeiros da Silva, Aline Abati Bolzan e Berta Maria Heinzmann*}

Departamento de Farmácia Industrial, Centro de Ciências da Saúde, Universidade Federal de Santa Maria, Campus Universitário, 97105-900 Santa Maria - RS, Brasil

Recebido em 20/7/05; aceito em 14/10/05; publicado na web em 5/5/06

PYRROLIZIDINE ALKALOIDS FROM Senecio SPECIES. Senecio species contain a large variety of secondary metabolites and many of these plants afford pyrrolizidine alkaloids. This paper is a review of the literature, describing 62 pyrrolizidine alkaloids already isolated in 62 of more than 2000 species of Senecio, distributed worldwide. The structure-activity relationships involving their toxicity are also discussed, since some Senecio species used for medicinal purposes are responsible for causing serious adverse effects.

Keywords: Senecio; pyrrolizidine alkaloids; hepatotoxicity.

\section{INTRODUÇÃO}

O gênero Senecio (tribo Senecioneae, Asteraceae) é constituído por mais de 2000 espécies de ampla distribuição mundial ${ }^{1}$. No Brasil, foram catalogadas cerca de 85 espécies pertencentes ao gênero, dentre as quais 33 são nativas da região sul ${ }^{1}$ e 25 foram identificadas no Rio Grande do $\mathrm{Sul}^{2}$.

Os alcalóides pirrolizidínicos (APs) são considerados um importante grupo de constituintes do gênero Senecio. São conhecidos por causarem intoxicações em animais herbívoros de grande porte, como bovinos, ovinos, suínos e eqüinos, levando a perdas consideráveis na pecuária para o sul do Brasil, Argentina, Paraguai e Uruguai $^{3-5}$. A literatura também relata a ocorrência de intoxicações fatais em seres humanos devido ao consumo de espécies de Senecio contendo $\mathrm{Aps}^{6-8}$, que têm sido empregadas na medicina popular de diversos países, inclusive na América Latina ${ }^{9-11}$.

\section{TIPOS DE ALCALÓIDES PIRROLIZIDÍNICOS ENCONTRADOS EM ESPÉCIES DE Senecio}

APs são ésteres de aminoálcoois com um núcleo pirrolizidínico (necina) e ácidos alifáticos (ácidos nécicos), que podem ocorrer na forma de mono, di e diésteres cíclicos. As necinas caracterizam-se por apresentar um sistema bicíclico com um nitrogênio terciário como "cabeça de ponte", um grupamento hidroximetila em $\mathrm{C} 1$ e uma hidroxila em $\mathrm{C}^{8}$ (Figura 1). Os APs podem apresentar a necina insaturada entre os carbonos $\mathrm{C} 1$ e $\mathrm{C} 2$, sendo esta característica um pré-requisito para a sua toxicidade aguda e crônica ${ }^{8,12,13}$. Os APs que possuem a necina saturada não são tóxicos aos mamíferos ${ }^{14}$.

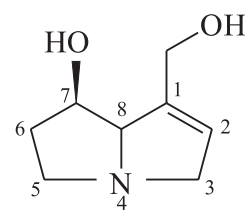

Figura 1. Estrutura básica de uma necina

*e-mail: hberta@ccs.ufsm.br
Segundo Trigo ${ }^{15}$, por serem muito comuns ao gênero, os APs podem ser utilizados como marcadores quimiossistemáticos para a tribo Senecioneae, uma vez que a maioria apresenta uma estrutura macrocíclica diéster do tipo senecionina (Tabela 1) e/ou são mono e diésteres do tipo triangularina (Tabela 2).

Embora os alcalóides pirrolizidínicos sejam considerados metabólitos secundários característicos do gênero Senecio, eles não estão presentes em todas as espécies e também têm sido relatados em gêneros vizinhos ${ }^{53}$. Além da variabilidade inter-específica na composição de APs, pode ocorrer variação intra-específica na concentração dos mesmos, conforme a época e o local da coleta, a parte da planta e seu quimiotipo ${ }^{24}$.

A biossíntese dos APs tem início nas raízes de Senecio, onde são produzidos primeiramente $\mathrm{N}$-óxidos da senecionina. Estes são transportados para os órgãos superiores da planta, onde sofrem alterações estruturais, originando os diferentes $\mathrm{APs}^{24}$. Entretanto, somente se tornam tóxicos quando metabolisados pelo fígado a uma forma pirrólica altamente reativa, conhecida como deidroalcalóide ${ }^{8}$.

O efeito hepatotóxico destes alcalóides, devido à atuação de seus metabólitos como agentes alquilantes, está bem estabelecido. Primeiramente ocorre uma oxidação (desidrogenação) no carbono- $\alpha$ ao $\mathrm{N}$, catalisada por monooxigenases do citocromo P- $450^{54}$. Os derivados pirrólicos assim originados são reativos e sofrem conversão espontânea, originando agentes eletrofílicos, que reagem com substâncias celulares de caráter nucleofílico através de uma adição de Michael. A glutationa reduzida apresenta caráter nucleofílico e, devido a esta característica, protege o organismo, uma vez que captura os derivados pirrólicos tóxicos, sendo esta a principal rota de detoxificação utilizada pelo organismo. No entanto, outros nucleofílicos das células, como ácidos nucleicos e proteínas vitais, também reagem com os derivados pirrólicos, formando adutos ${ }^{55}$. A alteração na estrutura de moléculas vitais leva à alteração de sua função, o que explica as diversas manifestações patológicas ocasionadas pelos APs.

As diferentes espécies animais apresentam suscetibilidades variáveis frente aos APs, na dependência do tipo de metabolismo enzimático destes no microssoma hepático. A produção do núcleo pirrólico em baixas quantidades e as altas taxas de conjugação com a glutationa parecem ser as razões para a maior resistência de ovelhas e hamsters à toxicidade destes alcalóides ${ }^{54}$. A ingestão crônica 
Tabela 1. Principais alcalóides pirrolizidínicos do tipo senecionina presentes em espécies do gênero Senecio

\begin{tabular}{lll}
\hline Alcalóides & Espécies de Senecio & Ref. \\
Pirrolizidínicos & & \\
\hline
\end{tabular}

\begin{tabular}{lll}
\hline Alcalóides & Espécies de Senecio \\
Pirrolizidínicos & & \\
\hline
\end{tabular}<smiles>C/C=C(/CC1(C)OC1(C)C(=O)OCC1=CCN2CCC1C2)C(=O)O</smiles>

S. jacobaea L.

16<smiles>C/C1=C/C(=O)OC2CCN3CCC(C1)C23</smiles>

S. cacaliaster Lam.

Acetilerucifolina

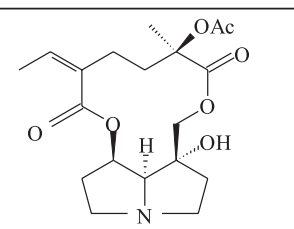

S. hadiensis Forsk. 17

12-O-acetil-hadiensina

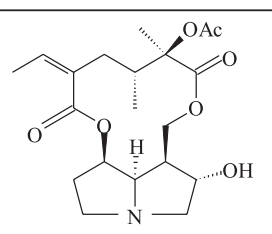

S. hadiensis Forsk

17

S. syringifolius O. Hoffm.

17

12-O-acetilrosmarinina

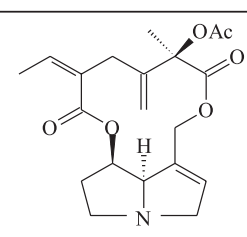

S. pterophorus DC.

7

Acetilseneciofilina

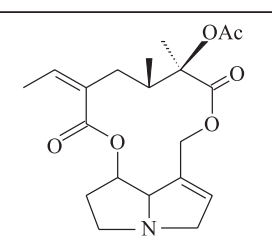

S. lautus Forster f. ex Willd. 18

S. magnificus F. Muell.

S. quadridentatus Labill.

19

19

Acetilsenecionin

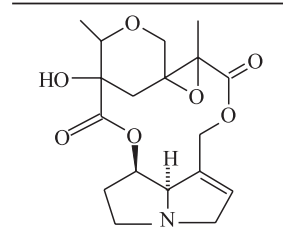

S. adonidifolius Loisel.

20

\section{N-óxido de \\ Erucifolina}

S. leptolobus DC.<smiles>COC1(C)CN(C)CCC=C1C(O)C(CCCN)OC(=O)C(C)C(C)Cl</smiles><smiles>C=C(CC(CC)C(=O)OCC1CCN2CCC1C(=O)OC2C)C(=O)O</smiles>

S. microphyllus Phil.

8

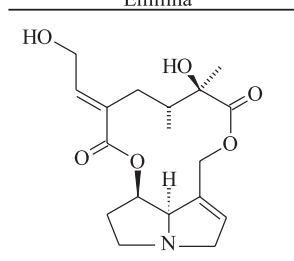

S. erucifolius L.

16

$S$. jacobaea L.

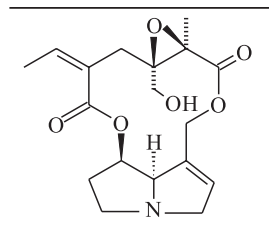

Erucifolina

\section{S. aquaticus Hill.}

S. argunensis Turcz.

S. erraticus Bertol.

S. erucifolius L.

S. jacobaea L.

S. persoonii De Not.

S. persoonii De Not.

27
Adonifolina<smiles>C=C(CC)C/C(=C/C)C(=O)OC1CCN2CC(O)C(COC(=O)C(C)(O)C(=O)O)C12</smiles>

S. syringifolius $O$. Hoffm.

17

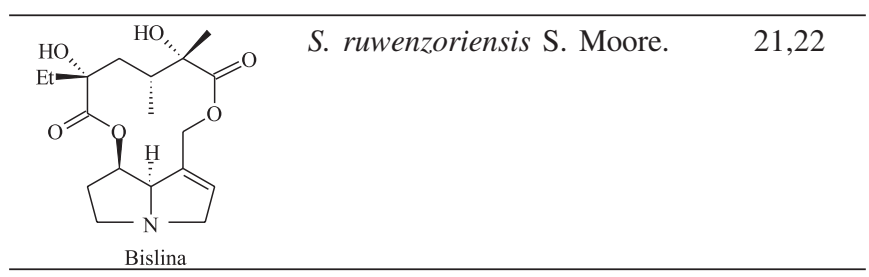


Tabela 1. continuação

\begin{tabular}{lll}
\hline Alcalóides & Espécies de Senecio & Ref. \\
Pirrolizidínicos & \\
\hline
\end{tabular}

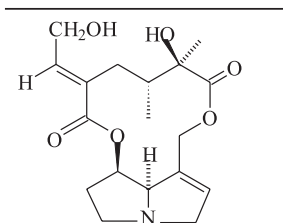

S. argunensis Turcz.

26

21-Hidroxi-integerrimina

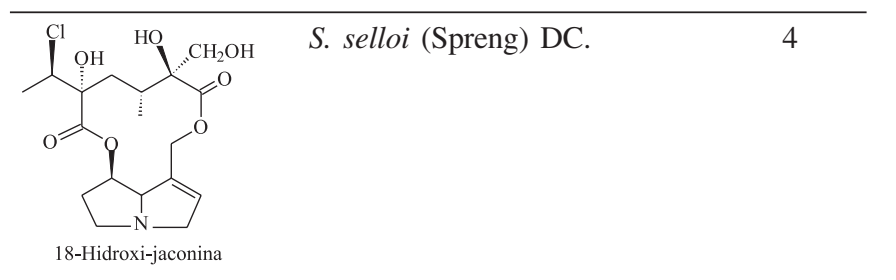

S. argunensis Turcz.

S. brasiliensis

(Sprengel) Less.

S. erucifolius L.

S. glabellus Poir.

S. heterotrichus DC.

S. jacobaea L.

S. leptolobus DC.

S. longilobus Benth.

S. malacitanus L.

S. murorum Remy
26

$4,10,15,31$

16

32

3,4

$16,24,33$

3,4

32

34

8

\begin{tabular}{lll}
\hline Alcalóides & Espécies de Senecio \\
Pirrolizidínicos & & Ref. \\
\hline
\end{tabular}<smiles>CC(O)C(O)C(CCC(C)(O)C(=O)OCC1=CCN2CCCC12)C(=O)O</smiles>

S. jacobaea L.

24,33

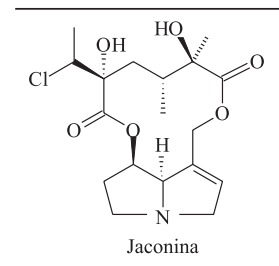

S. jacobaea L.

$16,24,33$

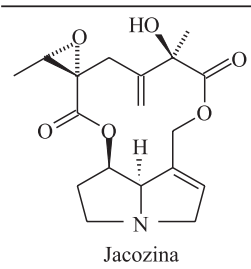

S. jacobaea L.

$16,24,33$

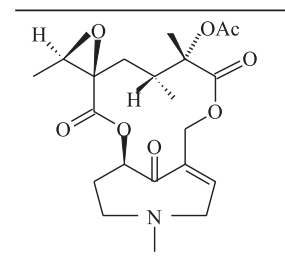

S. gallicus Vill.

S. microphyllus Phil.

28

8

Ligularizina<smiles>C/C=C1/CC[C@@](C)(O)C(=O)OCC2=CCN(CCC[C@H]2OC(=O)O)C1</smiles>

S. grandifolius Less.

S. leptolobus DC.

Neosenquirquina

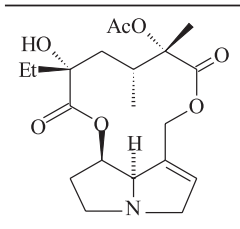

Isolina

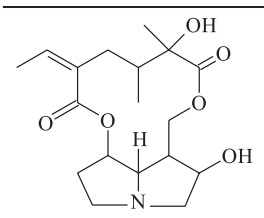

Isorosmarinina

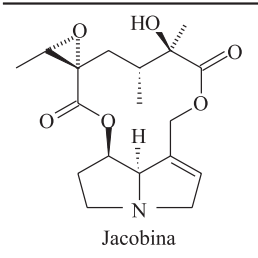

S. ruwenzoriensis S. Moore. 21,22

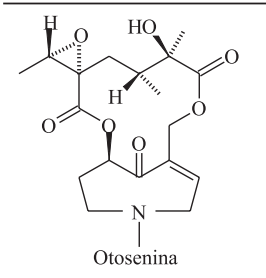

S. argunensis Turcz.

S. erraticus Bertol.

S. glaber Less.

S. leptolobus DC.

S. lorenthii Hochst.

26

8

8

4

36

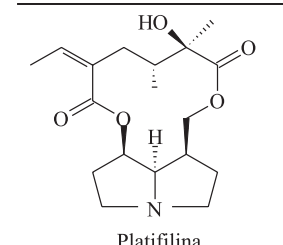

S. bonariensis Hook. et Arn.

37

S. granatensis Boiss.

S. jacalensis Greenm.

S. madrensis A. Gray

S. polypodioides Greene

38

39

39

Platifilina

S. jacobaea L.

$16,24,33$

N-óxido de platifilina

S. granatensis Boiss.

S. madrensis A. Gray

S. polypodioides Greene 
Tabela 1. continuação

\begin{tabular}{|c|c|c|}
\hline $\begin{array}{l}\text { Alcalóides } \\
\text { Pirrolizidínicos }\end{array}$ & Espécies de Senecio & Ref. \\
\hline Z-Retrorsina & $\begin{array}{l}\text { S. bonariensis Hook. et Arn. } \\
\text { S. brasiliens (Sprengel) Less. } \\
\text { S. cisplatinus Cabrera } \\
\text { S. conyzifolius Baker } \\
\text { S. cymbalorioides Nutt. } \\
\text { S. gilliesianus Hieron. } \\
\text { S. heterotrichus DC. } \\
\text { S. jacobaea } \text { L. } \\
\text { S. leptolobus DC. } \\
\text { S. longilobus Benth. } \\
\text { S. malacitanus L. } \\
\text { S. microphyllus Phil. } \\
\text { S. oleosus Vell. } \\
\text { S. othonnaeflorus DC. } \\
\text { S. oxiphyllus DC. } \\
\text { S. prionopterus B. L. } \\
\text { Rob. e Greenm. } \\
\text { S. pseudaureus Rydb. } \\
\text { S. selloi (Spreng) DC. } \\
\text { S. streptanthifolios Greene } \\
\text { S. uspallatensis Hook et Arn. }\end{array}$ & $\begin{array}{c}40 \\
4,10,15,31 \\
3,4 \\
37 \\
41 \\
42 \\
3,4 \\
16 \\
4 \\
32 \\
34 \\
8 \\
37 \\
43 \\
4 \\
39 \\
41 \\
3,4 \\
41 \\
44\end{array}$ \\
\hline
\end{tabular}

\begin{tabular}{llc} 
Alcalóides & Espécies de Senecio & Ref. \\
Pirrolizidínicos & & \\
\hline & S. aquaticus Hill. & 25 \\
& S. argunensis Turcz. & 26 \\
& S. brasiliensis & 10 \\
& (Sprengel) Less. & 45 \\
& S. crassiflorus (Poir.) DC. & 8 \\
& S. erraticus Bertol. & 16 \\
& S. erucifolius L. & 29 \\
& S. jacobaea L. & 32 \\
& S. laricifolius H.B.K. & 36 \\
& S. longilobus Benth. & 8 \\
& S. lorenthii Hochst. & 29 \\
& S. microphyllus Phil. & 4 \\
& S. multivenius Benth. & \\
& in Oerst. & \\
& S. oxiphyllus DC. & \\
& S. patagonicus Phil. & \\
& S. persoonii De Not. &
\end{tabular}

$\mathrm{N}$-óxido de

Retrosina

(Isatidina)
S. othonnaeflorus DC.

43

43

\section{N-óxido de} Seneciofilina
S. crassiflorus (Poir.) DC.

S. persoonii De Not.
45 27
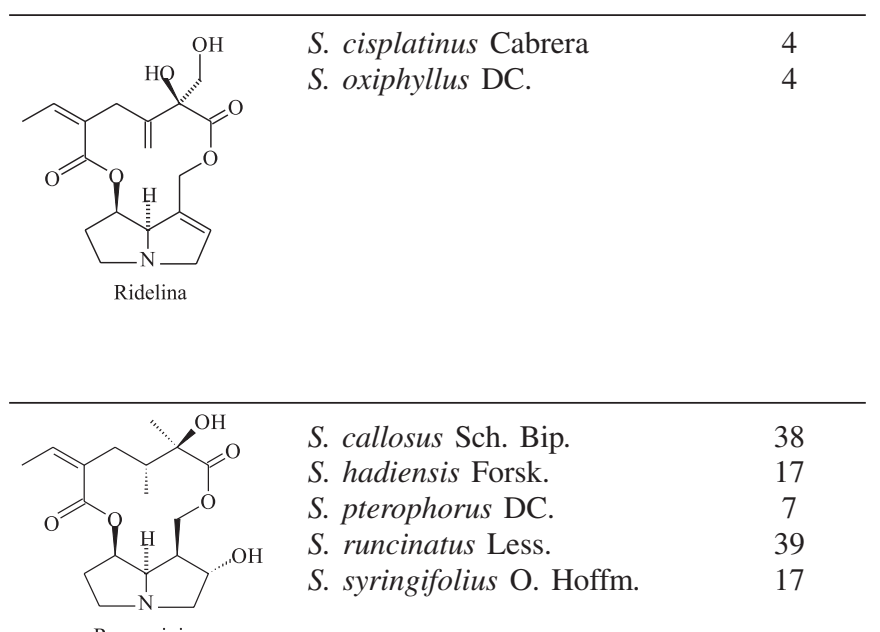

S. callosus Sch. Bip.

38

$S$. hadiensis Forsk.

S. pterophorus DC.

$S$. runcinatus Less.

S. syringifolius O. Hoffm.

Rosmarinina

N-óxido de

S. runcinatus Less.

39

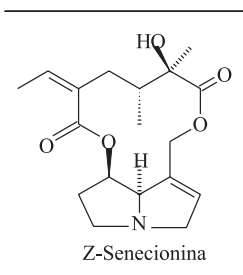

S. argunensis Turcz.

$S$. bonariensis Hook. et Arn.

S. brasiliens (Sprengel) Less.

$S$. cisplatinus Cabrera

S. crassiflorus (Poir.) DC.

$S$. cymbalorioides Nutt.

S. erraticus Bertol.

S. erucifolius L.

S. gallicus Vill.

S. gilliesianus Hieron.

S. glabellus Poir.

$S$. heterotrichus DC.

S. jacalensis Geenm.

$S$. jacobaea L.

S. laricifolius H.B.K.

S. leptolobus DC.

S. longilobus Benth.

S. madrensis A. Gray

S. malacitanus L.

S. microphyllus Phil.

$S$. multivenius Benth.

in Oerst.

S. nemorensis L.

S. oleosus Vell.

S. patagonicus Phil.

S. prionopterus B. L. Rob. e

Greenm.

S. pseudaureus Rydb.

S. pterophorus DC.

S. selloi (Spreng) DC.

S. streptanthifolios Greene

$S$. triangularis Hook.

\section{6}

37,40

$4,10,15$

3

45

41

8

16

28

42

32

4

38

$16,24,33$

29

4

32

39

34

8

29

30

37

8

39

41

7

3,4

41

41 
Tabela 1. continuação

\begin{tabular}{|c|c|c|c|c|c|}
\hline $\begin{array}{l}\text { Alcalóides } \\
\text { Pirrolizidínicos }\end{array}$ & Espécies de Senecio & Ref. & $\begin{array}{l}\text { Alcalóides } \\
\text { Pirrolizidínicos }\end{array}$ & Espécies de Senecio & Ref. \\
\hline $\begin{array}{l}\text { N-óxido de } \\
\text { Senecionina }\end{array}$ & $\begin{array}{l}\text { S. crassiflorus (Poir.) DC. } \\
\text { S. gallicus Vill. }\end{array}$ & $\begin{array}{l}45 \\
28\end{array}$ & E-Spartioidina & S. pterophorus DC. & 7 \\
\hline Senecivernina & $\begin{array}{l}\text { S. jacobaea L. } \\
\text { S. leptolobus DC. } \\
\text { S. selloi (Spreng) DC. }\end{array}$ & $\begin{array}{c}24 \\
4 \\
4\end{array}$ & E-Usaramina & $\begin{array}{l}\text { S. brasiliensis } \\
\text { (Sprengel) Less. } \\
\text { S. cisplatinus Cabrera } \\
\text { S. leptolobus DC. } \\
\text { S. malacitanus L. } \\
\text { S. murorum Remy. } \\
\text { S. oxiphyllus DC. } \\
\text { S. selloi (Spreng) DC. }\end{array}$ & $\begin{array}{c}4,10,15 \\
4 \\
4 \\
34 \\
8 \\
4 \\
4\end{array}$ \\
\hline Senquirquina & $\begin{array}{l}\text { S. gallicus Vill. } \\
\text { S. grandifolius Less. } \\
\text { S. laricifolius H.B.K. } \\
\text { S. leptolobus DC. } \\
\text { S. quebradensis Grenm. }\end{array}$ & $\begin{array}{c}28 \\
29 \\
29 \\
4 \\
29\end{array}$ & Uspalatina & $\begin{array}{l}\text { S. patagonicus Phil. } \\
\text { S. uspallatensis Hook et Arn. }\end{array}$ & $\begin{array}{c}8 \\
44\end{array}$ \\
\hline
\end{tabular}

Tabela 2. Principais alcalóides pirrolizidínicos do tipo triangularina presentes em espécies do gênero Senecio

\begin{tabular}{lll}
\hline Alcalóides & Espécies de Senecio & Ref. \\
Pirrolizidínicos &
\end{tabular}

9-Angelil-hastanecina

\begin{tabular}{lll}
$\begin{array}{l}\text { Alcalóides } \\
\text { Pirrolizidínicos }\end{array}$ & Espécies de Senecio \\
\hline
\end{tabular}

7-Angelil-platinecina

\begin{tabular}{lll}
\hline S. chrysocoma Meerb. & 49 \\
S-Angelil-platinecina &
\end{tabular}

S. schweinfurthii O. Hoffm.

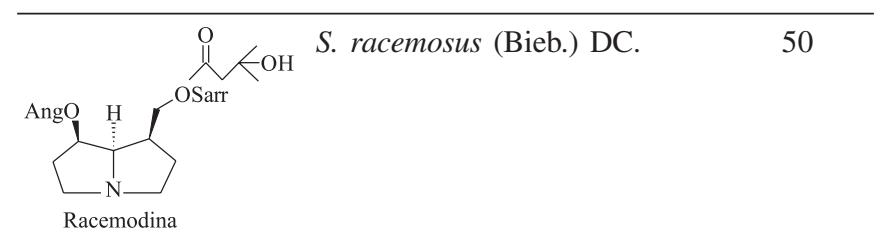


Tabela 2. continuação

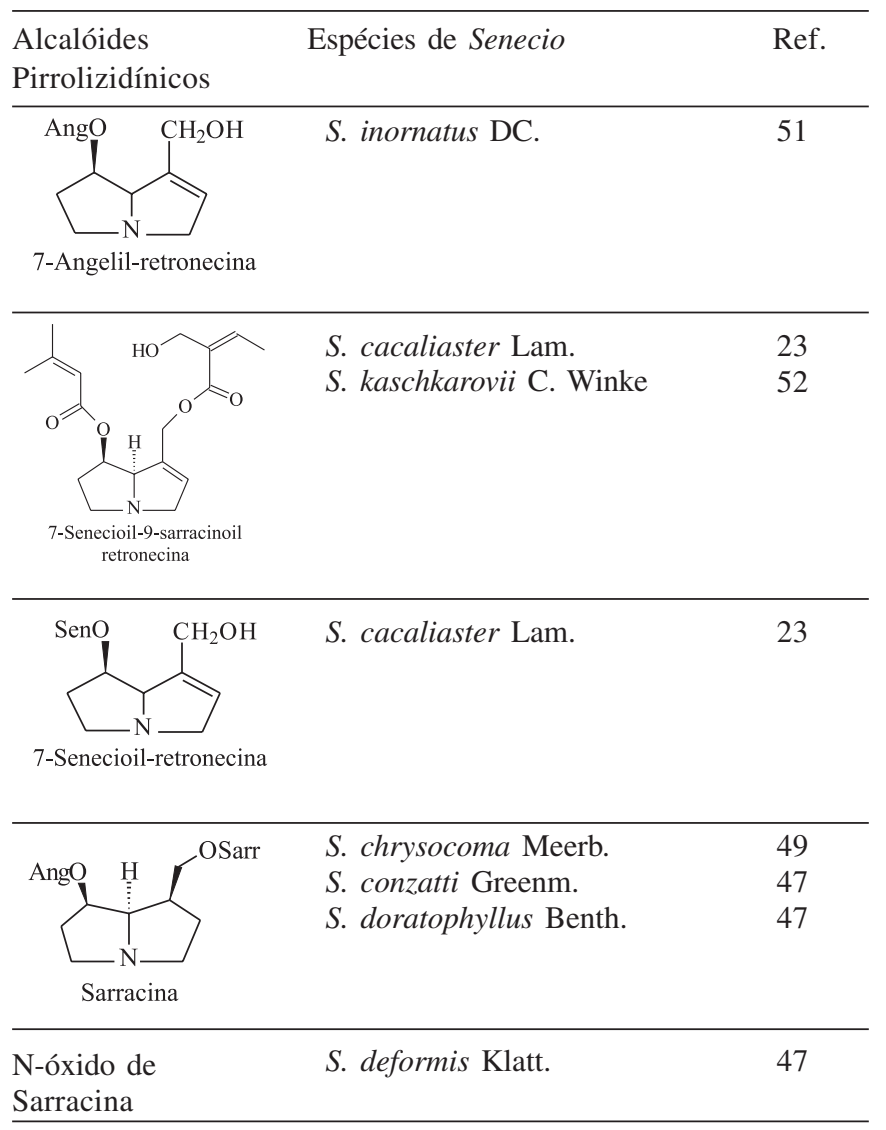

de plantas contendo APs, por animais de laboratório, levou ao desenvolvimento de câncer e, paralelamente, metabólitos de alguns APs mostraram atividade mutagênica in vitro ${ }^{56}$. No entanto, até o momento, não foi encontrada nenhuma correlação entre a exposição de humanos aos APs e o desenvolvimento de câncer. A análise de vários relatos da literatura sobre a exposição de seres humanos aos APs levou Parkash ${ }^{55}$ e colaboradores à conclusão de que estes compostos não são carcinogênicos aos seres humanos; entretanto, a exposição a estas substâncias pode causar doenças veno-oclusivas e cirrose infantil, responsáveis por vários casos de óbito.

\section{AGRADECIMENTOS}

\section{Ao CNPq e à CAPES.}

\section{REFERÊNCIAS}

1. Cabrera, A. L.; Klein, R. M. Em Flora Ilustrada Catarinense; Reitz, P. R., ed; Herbário Barbosa Rodrigues: Itajaí, 1975.

2. Matzenbacher, N. I.; Tese de Doutorado, Universidade Federal do Rio Grande do Sul, Brasil, 1998.

3. Habermehl, G. G.; Martz, W.; Tokarnia, C. H.; Dobereiner, J.; Mendez, M. C.; Toxicon 1988, 26, 275.

4. Krebs, H. C.; Carl, T.; Habermehl, G. G.; Phytochemistry 1996, 43, 1227.

5. Ilha, M. R. S.; Loretti, A. P.; Barros, S. S.; Barros, C. S. L.; Pesq. Veter. Brasil. 2001, 21, 123.

6. Fox, D. W.; Hart, M. C.; Bergeson, P. S.; Jarret, P. B.; Stillman, A. E.; Huxtable, R. J.; J. Pediatr. 1978, 93, 980

7. Liddell, J. R.; Logie, C. G.; Phytochemistry 1993, 34, 1629.

8. Villarroel, L. V.; Torres, R. G.; Urzúa, A.; Modak, B.; Henriquez, J.; Salgado, I.; Rev. Latinamer. Quim. 1997, 25, 109.

9. Garcia, V. M. N.; Gonzalez, A.; Fuentes, M.; Aviles, M.; Rios, M. Y.; Zepeda, G.; Rojas, M. G.; J. Ethnopharmacol. 2003, 87, 85.

10. Toma, W.; Trigo, J. R.; De Paula, A. C. B.; Brito, A. R. M. S.; J. Ethnopharmacol. 2004, 95, 345.

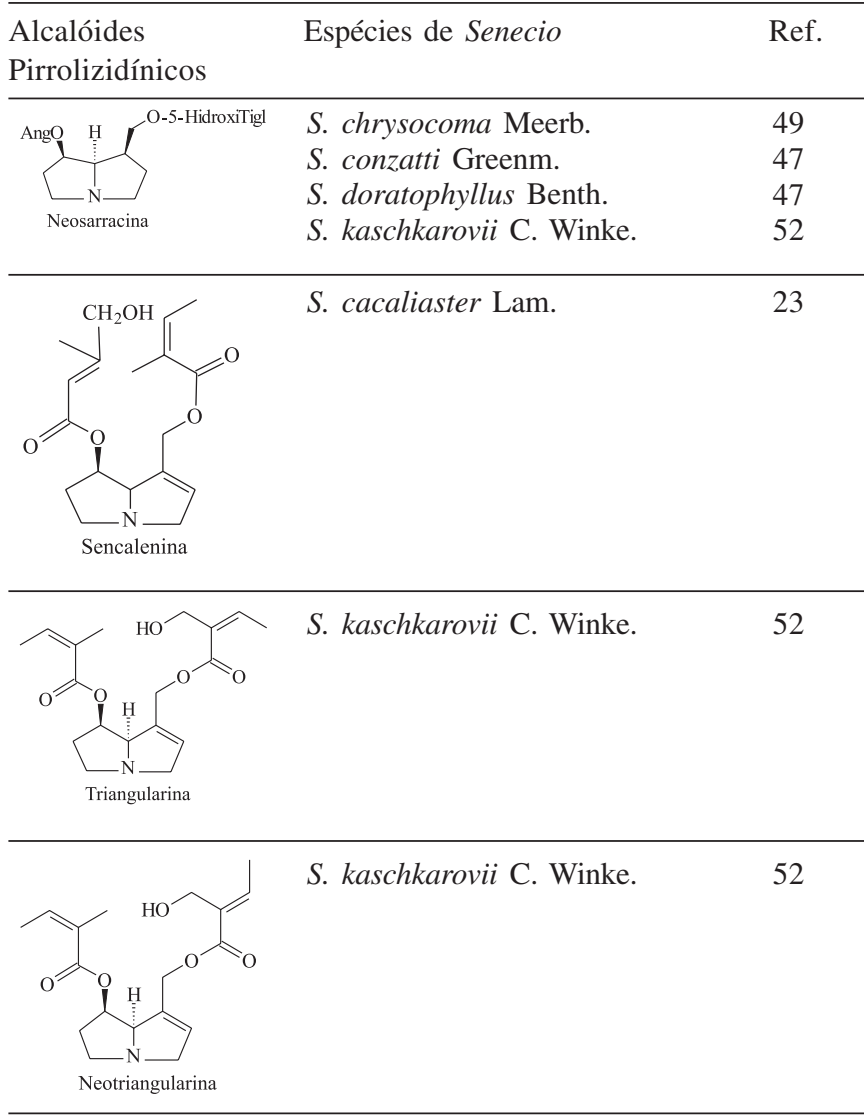

11. Bourdy, G.; Chavez de Michel, L. R.; Roca-Coulthard, A.; J. Ethnopharmacol. 2004, 91, 189.

12. Mattocks, A. R.; Driver, H. E.; Barbour, R. H.; Chem. Biol. Interact. 1986, $58,95$.

13. Jares, E. A.; Pomílio, A. B.; J. High Resol. Chromat. 1989, 12, 565.

14. Mattocks, A. R.; apud Biochem. Syst. Ecol. 1989, 30, 981.

15. Trigo, J. R.; Leal, I. R.; Matzenbacher, N. I.; Lewinsohn, T. M.; Biochem. Syst. Ecol. 2003, 31, 1011.

16. Witte, L.; Ernst, L.; Adam, H.; Hartmann, T.; Phytochemistry 1992, 31, 559.

17. Were, O.; Benn, M.; Munavu, R. M.; Phytochemistry 1993, 32, 1595.

18. Bohlmann, F.; Ziesche, J.; King, R. M.; Robinson, H.; Phytochemistry 1980, $19,2675$.

19. Zdero, C.; Bohlmann, F.; King, R. M.; Haegi, L.; Phytochemistry 1990, $29,509$.

20. Witte, L.; Ernst, L.; Wray, V.; Hartmann, T.; Phytochemistry 1992, 31, 1027.

21. Benn, M.; Were, O.; Phytochemistry 1992, 31, 3295.

22. Susag, L.; Parvez, M.; Mathenge, S.; Benn, M. H.; Phytochemistry 2000, $54,933$.

23. Roeder, E.; Wiedenfeld, H.; Kirstgen, R. B.; Phytochemistry 1984, 23, 1761.

24. Macel, M.; Vrieling, K.; Klinkhamer, P. G. L.; Phytochemistry 2004, 65, 865 .

25. Christov, V. S.; Mikhova, B. P.; Evstatieva, L. N.; Fitoterapia 2002, 73, 171.

26. Liu, K.; Roder, E.; Phytochemistry 1991, 30, 1303.

27. Roeder, E.; Bourauel, T.; Kersten, R.; Phytochemistry 1993, 32, 1051.

28. Urones, J. G.; Barcala, P. B.; Marcos, I. S.; Moro, R. F.; Esteban, M. L.; Rodriguez, A. F.; Phytochemistry 1988, 27, 1507.

29. Bohlmann, F; Zdero, C; Jakupovic, J.; Grenz, M.; Castro, V.; King, R. M.; Robinson, H.; Vincent, L. P. D.; Phytochemistry 1986, 25, 1151.

30. Wiedenfeld, H.; Röder, E.; Phytochemistry 1979, 18, 1083.

31. Santos-Mello, R; Deimlimg L. I.; Lauer Júnior, C. M.; Almeida, A.; Mutat. Res. 2002, 516, 23.

32. Ray, A. C.; Williams, H. J.; Reagor, J. C.; Phytochemistry 1987, 26, 2431.

33. Vrieling, K.; Derridj, S.; Phytochemistry 2003, 64, 1223.

34. Suau, R.; Cabezudo, B.; Rico, R.; Nájera, F.; López-Romero, J. M.; García, A.I.; Biochem. Syst. Ecol. 2002, 30, 981. 
35. Martz, W.; Habermehl, G. G.; Planta Med. 1986, 52, 503

36. Noorwala, M.; Mohammad, F. V.; Ahmad, V. U.; Sener, B.; Ergun, F.; Deliorman, D.; Fitoterapia 2000, 71, 618.

37. Paiva, J. A.; Barata, L. E. S.; Trigo, J. R.; Biochem. Syst. Ecol. 2004, 32, 1219.

38. Romo de Vivar, A.; Pérez, A. L.; Vidales, P.; Nieto, D. A.; Villaseñor, J L.; Biochem. Syst. Ecol. 1996, 24, 175.

39. Pérez-Castorena, A. L; Arciniegas, A.; Martinez, F.; Villasenõr, J. L.; Romo de Vivar, A.; Biochem. Syst. Ecol. 2000, 28, 279.

40. Tettamanzi, M. C.; Jares, E. A.; Pomilio, A. B.; Fitoterapia 1992, LXIII, 551.

41. Bai, Y.; Benn, M.; Majak, W.; Planta Med. 1996, 62, 71.

42. Guidugli, F. H.; Pestchanker, M. J.; De Salmeron, M. S. A.; Giordano, O. S.; Phytochemistry 1986, 25, 1923.

43. Zdero, C.; Bohlmann, F.; Liddell, J. R.; Phytochemistry 1989, 28, 3532.

44. Pestchanker, M. J.; Ascheri, M. S.; Giordano, O. S.; Phytochemistry 1985, 24, 1622.

45. Tettamanzi, M. C.; Jares, E. A.; Iannone, L. M.; Pomilio, A. B.; Fitoterapia 1994, $L X V, 468$.
46. Liddell, J. R.; Logie, C. G.; Phytochemistry 1993, 34, 1198

47. Pérez-Castorena, A. L.; Arciniegas, A.; Villasenõr, J. L.; Romo de Vivar, A.; Biochem. Syst. Ecol. 1999, 27, 835.

48. Benn, M. H.; Mathenge, S.; Munavu, R. M.; Were, S. O.; Phytochemistry 1995, 40, 1327.

49. Grue, M. R.; Liddell, J. R.; Phytochemistry 1993, 33, 1517.

50. Ahmed, W.; Khan, A. Q.; Malik, A.; Ergun, F.; Sener, B.; Phytochemistry 1993, 32, 224

51. Wiedenfeld, H.; Roeder, E.; Luck, W.; Planta Med. 1996, 62, 483.

52. Cheng, D. L.; Niu, J. K.; Roeder, E.; Phytochemistry 1992, 31, 3671.

53. Bohlmann, F.; Zdero, C.; Jakupovic. J.; Misra, L. N.; Banerjee, S.; Singh, P.; Baruah, R. N.; Metwally, M. A.; Hirschmann, G. S.; Vincent, L. P. D.; King, R. M.; Robinson, H.; Phytochemistry 1985, 24, 1249.

54. Huan, J. Y.; Miranda, C. L.; Buhler, D. R.; Cheeke, P. R.; Toxicol. Lett. 1998, 99, 127

55. Parkash, A. S.; Pereira, T. N.; Reilly, P. E. B.; Seawright, A. A.; Mutat. Res. 1999, 443, 53.

56. Chojkier, M.; J. Hepatol. 2003, 39, 437. 